\title{
Cross-Species Integrative Functional Genomics in GeneWeaver Reveals a Role for Pafah1b1 in Altered Response to Alcohol
}

\author{
Jason A. Bubier ${ }^{1}$, Troy D. Wilcox ${ }^{1}$, Jeremy J. Jay ${ }^{1,2}$, Michael A. Langston ${ }^{3}$, Erich J. Baker ${ }^{4}$ \\ and Elissa J. Chesler ${ }^{1 *}$ \\ ${ }^{1}$ The Jackson Laboratory, Bar Harbor, ME, USA, ${ }^{2}$ Department of Bioinformatics and Genomics, The University of North \\ Carolina at Charlotte, North Carolina Research Campus, Kannapolis, NC, USA, ${ }^{3}$ Department of Electrical Engineering and \\ Computer Science, University of Tennessee, Knoxville, TN, USA, ${ }^{4}$ School of Engineering and Department of Computer \\ Science, Baylor University, Waco, TX, USA
}

OPEN ACCESS

Edited by:

Allan V. Kalueff,

ZENEREI Institute, USA; Guangdong

Ocean University, China; St

Petersburg State University, Russia

Reviewed by:

Mamiko Koshiba,

Saitama Medical University, Japan

Evan Joseph Kyzar,

University of Illinois at Chicago, USA

*Correspondence:

Elissa J. Chesler

elissa.chesler@jax.org

Received: 13 November 2015 Accepted: 04 January 2016

Published: 21 January 2016

Citation:

Bubier JA, Wilcox TD, Jay JJ,

Langston MA, Baker EJ and

Chesler EJ (2016) Cross-Species

Integrative Functional Genomics in

GeneWeaver Reveals a Role for

Pafah1b1 in Altered Response to

Alcohol. Front. Behav. Neurosci. 10:1

doi: 10.3389/fnbeh.2016.00001
Identifying the biological substrates of complex neurobehavioral traits such as alcohol dependency pose a tremendous challenge given the diverse model systems and phenotypic assessments used. To address this problem we have developed a platform for integrated analysis of high-throughput or genome-wide functional genomics studies. A wealth of such data exists, but it is often found in disparate, non-computable forms. Our interactive web-based software system, Gene Weaver (http://www.geneweaver.org), couples curated results from genomic studies to graph-theoretical tools for combinatorial analysis. Using this system we identified a gene underlying multiple alcohol-related phenotypes in four species. A search of over 60,000 gene sets in GeneWeaver's database revealed alcohol-related experimental results including genes identified in mouse genetic mapping studies, alcohol selected Drosophila lines, Rattus differential expression, and human alcoholic brains. We identified highly connected genes and compared these to genes currently annotated to alcohol-related behaviors and processes. The most highly connected gene not annotated to alcohol was Pafah $1 \mathrm{~b} 1$. Experimental validation using a Pafah 1 b1 conditional knock-out mouse confirmed that this gene is associated with an increased preference for alcohol and an altered thermoregulatory response to alcohol. Although this gene has not been previously implicated in alcohol-related behaviors, its function in various neural mechanisms makes a role in alcohol-related phenomena plausible. By making diverse cross-species functional genomics data readily computable, we were able to identify and confirm a novel alcohol-related gene that may have implications for alcohol use disorders and other effects of alcohol.

Keywords: behavioral genomics, combinatorial data integration, functional genomics

\section{INTRODUCTION}

With the expanding diversity of sequenced genomes and decreasing costs of functional genomic experiments, numerous large-scale genomic, transcriptomic, metabolomics, and proteomic studies have been performed. Public repositories and private data collections of functional genomic data provide a rich context for the discovery of pathways underlying biological processes 
conserved across species and experimental systems. Exploiting these data for discovery of disease related genes requires extensive harmonization of identifiers for biological molecules and scalable, integrative analysis strategies for aggregating and synthesizing data across diverse technologies, species, and experimental paradigms. To address this need, we developed a publicly available web-based software system, GeneWeaver.org, that combines curated and user submitted functional genomic data (i.e., gene sets consisting of genes and gene products) from diverse experiments across numerous species, harmonized through homology and identifier translation tables, and coupled to a suite of software tools for combinatorial integration and analysis of these diverse data (Baker et al., 2012). The software in GeneWeaver includes analysis tools for evaluation of gene set-gene set intersections and scalable graph algorithms for setset matching to prioritize genes and gene sets of interest. These tools are directly incorporated into GeneWeaver, allowing users to prioritize genes in real time based on the relations among user-selected and user-entered gene sets. This strategy enables users to find consensus among highly heterogeneous experiments implicating genes in disease.

To demonstrate the application of integrative analysis of genome-wide functional genomics experiments, we applied GeneWeaver to identify novel genes related to alcohol-use disorder. Alcohol-use disorders are a major public health problem; over 16 million US adults had an alcohol-use disorder in 2013 (SAMHSA, 2013), and globally alcohol misuse is the leading risk factor for premature death and disability among people ages 15 and 49 (Lim et al., 2012). Unfortunately, efforts to identify the causative biological and genetic factors, and thus to develop novel pharmacological therapies, are hindered by the complexity of the disorder. Many molecular entities encoded throughout the genome underlie the various facets of alcohol-use disorder. Investigators have used a variety of species and experimental strategies to identify behavioral and molecular responses to alcohol. Conserved pathways or orthologous genes have been implicated in alcohol-related phenotypes across species (Morozova et al., 2007; McGary et al., 2010). Each study has advantages afforded by specific strengths of the individual disease models and the genetic tools available to interrogate these models, each representing various facets of alcohol effects and the progression to alcohol-use disorders. Finding consensus among these results has the potential to reveal the central mechanisms of disease, dissociated from experimental idiosyncrasies. The tremendous breadth of biological data, diversity of models, and the complexity of matching multiple genes to multiple phenomena across species has made the discovery of convergent findings difficult. Furthermore, many of the genome-wide platforms only probe a susbest of biomolcular entities. In GeneWeaver analyses, we account for the lack of comprehensive coverage in diverse functional genomic platforms by evaluating only those features in the possible intersection of each pair of data sets. In the present study, we apply our novel approach that incorporates genome-wide functional genomics experiments to aggregate convergent evidence for the discovery of novel genealcohol-use disorder associations. Importantly, this approach has wide applicability for finding genes in many other disease areas.
Data from GeneWeaver were queried to find alcohol related studies and analyzed to identify highly connected genes, i.e., those found in multiple experimentally associated alcoholrelevant gene sets. These results were compared to rigorously curated and often widely studied genes from Online Mendelian Inheritance in Man (OMIM) (Amberger et al., 2015) that were previously associated with alcohol-use disorder in humans or to Mammalian Phenotype (MP) Ontology term (Smith and Eppig, 2009) annotations to alcohol-related phenotypes in mice (Bubier and Chesler, 2012). A single gene, Pafah1b1, was determined to be the most highly connected and was not yet previously associated with alcohol use disorder in the annotated resources. Utilizing an existing viable heterozygous knock-out in mice we validated a role for Pafah $1 b 1$ in several alcohol-related phenotypes. These results demonstrate the potential of integrative genomics to identify novel candidate genes for human diseases.

\section{MATERIALS AND METHODS}

\section{Integrative Genomics in GeneWeaver.Org Database}

GeneWeaver's database currently contains $\sim 75,000$ gene sets. Data have been curated as described in Baker et al. (2012). Briefly, each gene set is assigned a Tier. Tiers I, II, and III represent public resources, machine generated resources, and human curated data sets, respectively. Tiers IV and V represent data submissions from users that are either pending curatorial review or stored for private use. To find convergence of experimentally derived gene associations from genomewide experiments the query was restricted to Tier III and IV. The database was queried (Date: Aug 2011) for Tier III and IV alcohol-related gene sets from three major experiment types: (i) QTL candidate genes, (ii) GWAS candidates, and (iii) differential expression experiments. A query for "Alcohol or Alcoholism," followed by manual review omitting false positive search results, e.g., those for which alcohol was mentioned in the publication abstract but was not relevant to the specific gene set, resulted in the retrieval of 32 data sets.

\section{Hierarchical Similarity Graph}

The Hierarchical Similarity Graph tool in GeneWeaver is used to group experimentally derived gene-set results based on the genes they contain. For a collection of input gene sets, this tool presents a graph of hierarchical relationships in which each terminal node represents individual gene sets and each parent node represents gene-gene set bicliques found among combinations of these sets using the maximal biclique enumeration algorithm (MBEA) (Zhang et al., 2014). The resulting graph structure is determined solely from the gene-set intersections of every populated combination of gene sets. In terms of gene sets, the smallest intersections (fewest gene sets, most genes) are at the right-most levels, and the largest intersections (most gene sets, fewest genes) are at the left of the graph. To prune the hierarchical similarity graph, bootstrapping is performed. The graph in the present analysis was sampled with replacement at $75 \%$ for 1000 iterations; node-node parent-child relationships occurring in greater than $50 \%$ of the results were included in the bootstrapped graph. 


\section{GeneSet Graph}

The GeneSet Graph tool generates a bipartite graph visualization of genes and gene sets. GeneWeaver operates on graphs with two sets of vertices, where genes are represented in one partite set, and gene sets represented in the other. A degree threshold is applied on the gene partite set to reduce the graph size. In the gene-set graph visualization tool, low-degree gene vertices are displayed on the left, followed by the gene-set vertices. High-degree genes are displayed on the right, in increasing order of connectivity.

\section{Comparison to Known Alcohol-Related Genes}

Tier I data in GeneWeaver refers to gene sets from curated data obtained from major public resources including gene annotations to Mammalian Phenotype Ontology (MP) and Gene Ontology (GO), curated functional associations in Neuroinformatics Framework (Gardner et al., 2008), and curated chemicalgene interactions in the Comparative Toxicogenomics Database (Davis et al., 2013). These data comprise a source of "ground truth" validated associations from gene to biological constructs. Resource-grade data is updated on a 6-month cycle. A search of tier I resources for canonical genes associated with alcohol resulted in 52 gene sets. These were connected with MP terms (Smith and Eppig, 2009), or the Online Mendelian Inheritance in Man (OMIM) database (Amberger et al., 2015). The Boolean Algebra tool provides gene-set combinations by deriving new sets consisting of the union, intersection, or high-degree genes within a group of gene sets, i.e., those that are found in a number of gene sets exceeding user-defined thresholds. With this feature, large numbers of gene sets can be compared or collapsed into a smaller number of gene sets. Using (Baker et al., 2012) this tool, these gene sets were all merged to make a single set of "known alcohol genes."

\section{Emphasis Genes}

The known alcohol genes identified using the Boolean Algebra tools were designated as "emphasis genes." Nodes containing emphasis genes are highlighted on the Hierarchical Similarity Graph to identify those specific nodes that contain genes previously associated with human alcoholism or mouse alcoholrelated phenotypes. This analysis reveals that although many genes have been identified in specific studies of alcoholism, the most highly represented genes among the 32 data sets are not currently annotated to alcoholism. The highest node contains Pafah1b1, which was selected for candidate gene validation in this work.

\section{Experimental Validation of Pafah1b1 in Alcohol-Related Behavior Mice}

All work was approved by the Institutional Animal Care and Use Committee at The Jackson Laboratory (JAX). Experiments were performed during the light phase of the light:dark cycle. Mice were group-housed with pine shavings, Shepard shacks, and nesting materials. Mice were maintained in specific-pathogen free facilities with sterilized acidified ( $\mathrm{pH} 2.5-3)$ water and NIH31 5K52 chow.
To produce the heterozygous knockout mice, the floxed allele of Pafah1b1b (Pafah1b1 ${ }^{\text {tm } 2 A w b}$; JR\#008002 129SPafah1b1 ${ }^{t m 2 A w b / J}$ ) (Hirotsune et al., 1998) was crossed to male mice with germline expressing Cre (Protamine 1 promoter driven Cre; JR\#003328 129S/Sv-Tg(Prm-cre)58Og/J) (O’Gorman et al., 1997) mouse to produce $129 \mathrm{~S}-\mathrm{Pafah} 1 \mathrm{~b}^{\mathrm{tm} 2.2 \mathrm{Awb}} / \mathrm{Ejc}$. Two cohorts of adult heterozygous mice were tested in our testing protocol; cohort 1 included 18 heterozygous mutants (eight females and 10 males) and 22 WT mice (11 females and 11 males) which were tested at 18 weeks of age, and cohort 2 comprised 16 heterozygous mutants (10 females and six males) and 13 WT mice (10 females and three males) tested at both 18 weeks of age (hypothermia $2.25 \mathrm{~g} / \mathrm{kg}$ ) and again at 6-8 months of age (sufficiently removed from initial testing to avoid development of chronic tolerance).

\section{Overall Testing Procedure}

\section{General Behavioral Testing Procedures}

Mice were subjected to a brief battery of non-invasive behavioral tests to assess activity, anxiety, and alcohol preference (Hamre et al., 2007). Mice were habituated to the testing room for $1 \mathrm{~h}$ prior to testing. Two experimenters participated in the testing, and a single experimenter handled the mice for each test. The same individuals were in the room during all sessions of a particular test.

\section{Assessment of Alcohol Effects}

For cohort 1, on the first day naive mice were tested in the open field, rotarod, and light-dark box, and the baseline body temperature was taken. On day 2 the mice were injected with $1.25 \mathrm{~g} / \mathrm{kg}$ EtOH before performing the same series of assays. At $60 \mathrm{~min}$ post-injection their body temperature was taken, and blood was collected for determination of ethanol concentration. In behavioral testing, a multiple-test testing battery can introduce carry-over effects, whereby the experience of one assay interferes with subsequent assays. The order of the behavioral tests in this battery was based upon previous work (Hamre et al., 2007) that demonstrated differential responses to ethanol or differences in ethanol consumption in three standard inbred strains of mice. The control mice underwent the same testing battery, enabling us to determine the effect of the knockout on phenotype.

\section{Body Temperature}

Body temperature was recorded rectally using a digital thermometer (BIO-TK8851, EB Instruments, Pinellas Park, FL) with a B Ret-3 probe designed for mice (L: $19 \mathrm{~mm}$; diam: $1.8 \mathrm{~mm}$; Cable: $100 \mathrm{~cm}$ ). The difference between the pre-EtOH temperature and the post EtOH temperature was considered the hypothermic response. This approach was used on mice in both cohorts 1 and 2 .

\section{Accelerating Rotarod}

This approach was used on cohort 1 , mice that receive a nonsedative dose $(1.25 \mathrm{~g} / \mathrm{kg})$ of ethanol. For each trial, each mouse was placed in one chamber of the rotarod facing the back wall of the apparatus. The rod was spinning at zero revolutions per minute (r.p.m.) at the beginning of the test and accelerated to 20 
r.p.m at a rate of $1 \mathrm{rpm} / 6 \mathrm{~s}$. The 3 - in-diameter rod was covered with 320-grit automotive sandpaper (Rustay et al., 2003). The latency to fall was recorded. On day 1, each mouse was trained on 10 trials on the rotarod. Because the latency to fall generally stabilizes after five trials, the last three of the 10 trials were used to calculate the mean latency to fall. On day 2, for analysis of ethanol-induced ataxia, only three trials were given and all were used to compute a per- mouse average post-ethanol latency.

\section{Loss of Righting Reflex (LORR)}

This assay was performed only the second cohort of mice. This group received a sedative dose of $\mathrm{EtOH}(2.5 \mathrm{~g} / \mathrm{kg})$, and were assayed for hypothermia as well as LORR measured. The LORR test is used to measure the hypnotic/sedative effects of acute ethanol and produces two time measures: latency to loss of righting reflex and duration of loss of righting reflex (DLRR). Based on the original protocol (Crabbe et al., 1981) modified for increased sensitivity (Ponomarev and Crabbe, 2002), mice were weighed and then injected i.p with ethanol $(2.5 \mathrm{~g} / \mathrm{kg}, 20 \%$ EtOH v/v). Mice were placed in a cylindrical restrainer in an upright position (Stoeltinger Co., Wood Dale, IL). The restrainer is a hollow cylinder attached to a square base at one end and an adjustable lid/moveable disk at the other open end that is used to position the head. Once a mouse was placed in the restrainer, the horizontal restrainer was rolled 90 degrees to the right every 2$3 \mathrm{~s}$. For the first few iterations of this procedure, prior to the onset of the ethanol's effect, the mice immediately right themselves. After 10-20 of such tests, the mice tend to remain on their back (for $\sim 5 \mathrm{~s}$ ) after two successive 90-degree turns. The LORR was calculated as the time interval between the onset of injection and the end of the second successive failed righting attempt. The DLRR is recorded as the time between injection and return of the righting reflex. This was measured in 3-10-min intervals following i.p injection. Return of the righting reflex occurs if animals can right themselves from a supine position within a 5-s period or could not be placed on their back after eight successive 90 -degrees turns in the restrainer.

\section{Locomotor Activity in the Open Field}

The open-field arena was an opaque Plexiglas box $(39 \times 39 \times$ $39 \mathrm{~cm}$ ) with a dark gray floor, illuminated at $43 \pm 4$ lux in a $10 \times 15$ feet testing room. A $10 \times 10 \mathrm{~cm}$ center zone was defined for analysis. Each mouse was placed into the center of the arena and allowed to explore for $20 \mathrm{~min}$. Behavioral measures in the open field and light-dark box were recorded, including fecal boli in the open field and light-dark box and analyzed by real-time video tracking using Ethovision XT 8. The distance traveled in the first $4 \mathrm{~min}$ (locomotor activity response to novelty); total distance traveled (general locomotor activity); percentage of time in the center; and defecation/number of fecal-boli (anxiety-like behaviors) were obtained for each mouse before and after alcohol injection.

\section{Light-Dark Box}

The light-dark box comprised a box with an insert evenly dividing the open-field apparatus into light-dark compartments, with the light compartment illuminated at $17 \pm 2$ lux. Mice were placed into the dark compartment and a 20-min recording began when the lid was closed (Henderson et al., 2004). The following behaviors were measured percentage of time spent in the dark.

\section{Blood Ethanol Concentration (BEC)}

At the completion of alcohol-effect testing of cohort $1,1 \mathrm{~h}$ following ethanol injection, $30 \mu \mathrm{l}$ of serum was obtained by retroorbital bleeding. BEC was analyzed on a Beckman DXC clinical system.

\section{Alcohol Preference Testing}

Two-bottle free-choice methods were followed as previously described (Bachmanov et al., 2002; Mulligan et al., 2008). Individually housed mice from cohort 2 were presented with two tubes containing an ethanol solution (VWR International Radnor, PA) made in sterilized acidified ( $\mathrm{pH} 2.5-3)$ water or sterilized acidified water alone. The positions of the tubes were switched daily to avoid side preference effects. Sipper tubes were weighed daily in the middle of the light cycle, to the nearest $0.01 \mathrm{~g}$ to measure the consumption of each solution. For 8 days prior to testing, mice were habituated to single housing with multiple sipper tubes, each containing sterilized acidified water. The experimental period occurred over 20 days, during the first 4 days of which the mice received 3\% ethanol (v/v) and during the next 4 days of which they received $6 \%$ ethanol, followed by 4 days of each 9,12 , and $15 \%$ ethanol. Control bottles of ethanol and water were placed in an empty mouse box, weighed daily and their positions alternated. This weight was subtracted from daily results to account for loss in experimental samples due to handling and evaporation. The weight of the mouse after the protocol was used to calculate ethanol consumed and total consumption per $\mathrm{kg}$ body weight, there was weight data missing from six mice, which were excluded from these calculations but included in preference.

\section{Statistical Analysis}

All analyses of mutant experiments were conducted using JMP 10 (SAS Institute). Alcohol preference was calculated as the amount of alcohol consumed as a percentage of the total alcohol and water consumed for both $3 \%$ and $10 \% \mathrm{EtOH}$. The full model is

$$
\text { Preference }=\beta_{0} \text { Sex }+\beta_{1} \text { Genotype }+\beta_{2}(\text { Sex } \times \text { Genotype })+\varepsilon,
$$

where $\varepsilon$ is random error. The parameters $(\beta, \varepsilon)$ were estimated by Type III non-sequential ordinary least squares in the ANOVA model. Repeated measures analysis of variance was performed using MANOVA to estimate the effects of genotype, sex and their interaction on both pre- and post-treatment measures. In all cases, the full model was fit and reduced by dropping non-significant interactions followed by main effects.

For ethanol-induced hypothermia,

$$
\begin{aligned}
\text { Temperature }= & \beta_{0} \text { Sex }+\beta_{1} \text { Genotype }+\beta_{2}(\text { Sex } \times \text { Genotype }) \\
& +\underset{\sim}{\mathbf{U}}+\varepsilon,
\end{aligned}
$$

where $\varepsilon$ is random error. The parameters $(\beta, \varepsilon)$ were estimated by Type III non-sequential ordinary least squares in the ANOVA model. Repeated measures analysis of variance was performed 


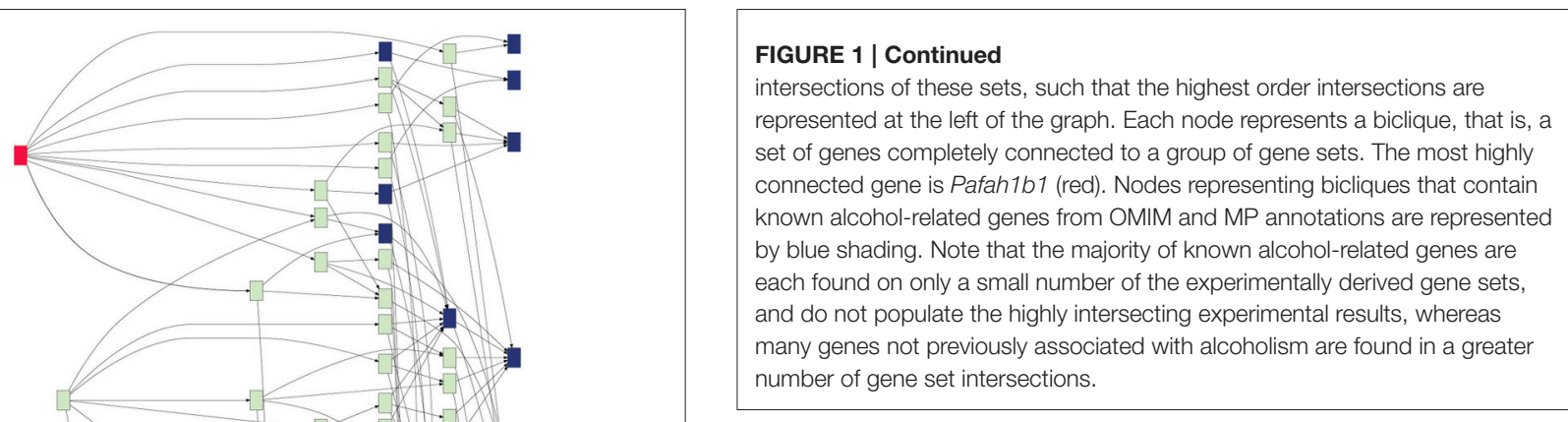

FIGURE 1 | Continued set of genes completely connected to a group of gene sets. The most highly connected gene is Pafah $1 b 1$ (red). Nodes representing bicliques that contain known alcohol-related genes from OMIM and MP annotations are represented and do not populate the highly intersecting experimental results, whereas many genes not previously associated with alcoholism are found in a greater fer intersections.

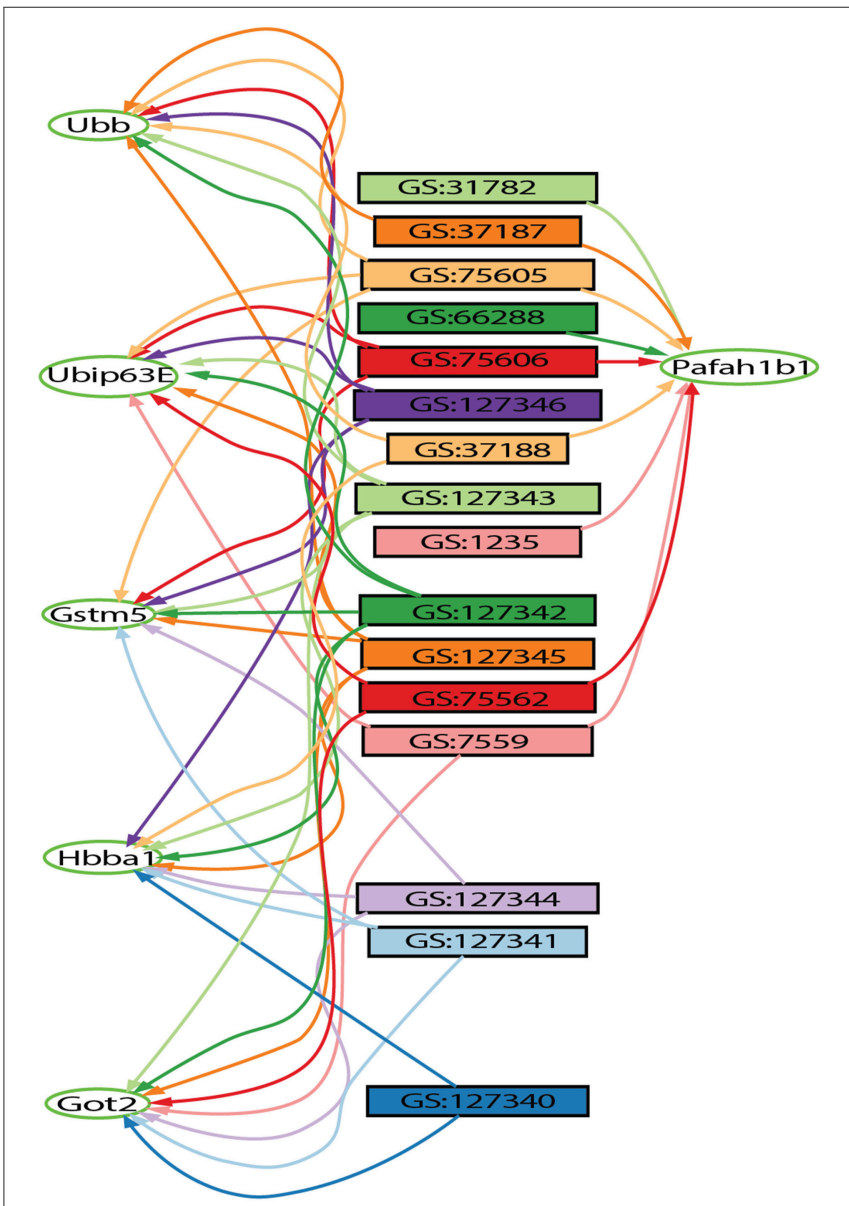

FIGURE 2 | Output from the gene set graph tool showing Pafah1b1-connected gene sets and other high-degree genes found on these sets. The genes are represented by oval shaped nodes, edges represent gene set membership, and the rectangular nodes represent gene sets retrieved from the GeneWeaver database. Other highly connected genes include Gstm5, Ubb, Hbaa1, Got2, and Ubi-p63E.

FIGURE 1 | Hierarchical similarity graph of experimentally derived alcohol-related genes sets. Terminal nodes, which are shown at the right-most level of the graph, represent the individual gene sets retrieved from the GeneWeaver database and each level above reflects higher order

(Continued) using MANOVA to estimate the effects of genotype, sex and their interaction. $\mathbf{U}$ represents the symmetric nature of the variance co-variance matrix of the random effects. In order to determine the nature of differences detected in the ANOVA model, planned contrasts were performed giving the terms not included in the model a weight of zero and giving the terms to be compared (sex and genotype) values of opposite weights, -1 and +1 . 
To adjust for the multiple testing of mice tested in cohorts 1 and 2, a false-discovery rate $q$-value was calculated using the $\mathrm{R}$ package qualue, setting fdr. level=0.05, pi0.method="bootstrap," $\operatorname{adj}=1.2$ (Storey and Tibshirani, 2003). Significant values were those with a familywise alpha $<0.05$.

\section{RESULTS}

\section{Integrative Functional Genomic Analyses}

GeneWeaver's database was queried for alcohol-related gene sets. The analysis was restricted to experimentally derived gene associations from genome-wide experiments across three major data types (differential expression, gene expression-phenotype correlations, and QTL positional candidates), which resulted in 32 gene sets (Table S1) from five species (Mus musculus, Rattus novregicus, Danio rerio, Drosophilia melanogaster, and Homo sapiens). GeneWeaver's Hierarchical Similarity Graph tool was used to perform a de novo aggregation of these 32 datasets based on the genes they contain (Figure 1). GeneWeaver represents the result as a bipartite graph. In such a graph, there are two disjoint sets of vertices, or nodes, with genes represented in one partite set, and gene set identifiers represented in the other. Genes and gene set identifiers (vertices) are connected by edges to represent gene set membership. The sets were harmonized to a common unique identifier for each cluster of gene identifiers defined by homology mapping. The union of all gene sets represented 13,609 genes across five species, with 6647 unique clusters of homologous genes, connected to gene sets by 10,684 edges. Briefly, this analysis enumerates all set-set intersections and represents them in a hierarchical graph (Figure 1) of the relations among the intersections, such that individual gene sets are represented at the right of the graph and successively higher-order intersections at the left. Each node in the hierarchical similarity graph represents a set of genes completely connected to a group of input gene-sets (Baker et al., 2009). This completely connected sub-graph of the bipartite graph is referred to as a biclique. The proportion of populated nodes gives an estimate of the cohesiveness of experimental results associated with a given concept. Fewer, larger combinations of gene sets, indicate similarity of experimental results related to the search term, whereas a greater number of small combinations indicate fragmentation of the results, and a less cohesive search term. Of the 8.5 billion possible intersections, 2297 of these were populated in the Hierarchical Similarity Graph, including 456 nodes representing intersections populated with more than two genes. To prune the Hierarchical Similarity graph we applied bootstrapping, which sampled $75 \%$ of gene set members and generated graphs for 1000 iterations. Only nodes and edges found in more than $50 \%$ of bootstraps were retained. The resulting bootstrapped graph (Figure 1, Figure S1) represents these robust gene set intersections and contains 195 intersection nodes and 543 edges.

The Hierarchical Similarity Graph was then highlighted using a derived gene set of known alcohol related genes. This set includes genes that had either been annotated to an alcohol related MP terms (Smith and Eppig, 2009), or annotated in the Online Mendelian Inheritance in Man (OMIM) database
(Amberger et al., 2015) to alcohol-use disorders. This analysis reveals that although many genes have been identified in individual studies of alcoholism, the most highly represented genes among the 32 gene sets are not currently annotated to alcoholism. The intersections containing these genes were at most only four levels above the terminal nodes of the hierarchical similarity graph. The left five levels of the hierarchical similarity graph contained no genes previously annotated to alcohol or alcoholism. The left two levels, a total of six nodes, represent six distinct genes currently not associated with alcohol in single gene studies, but found at the intersection of multiple genome-wide experiments. The Hierarchical Similarity Graph comprises a data-driven organization of experiments with different regions potentially representing categorically different subsets of experimental results. Examination of the gene sets associated with the most highly connected genes (Figure S1), reveals regions or subspaces of the graph that represent species separation, for example, experiments in fly and human implicate genes not found in the overlap of mouse and fly experiments. Another observation is that there are regions of the graph that represent overlap of gene sets from the same experimental study. The gene in the highest level node of the hierarchical similarity graph was Pafah1b1 (Figure 2), platelet-activating factor acetylhydrolase, isoform $1 \mathrm{~b}$, subunit 1 , also known as Lis-1, for lissencephaly, type 1 . This gene was present among $28 \%$ of sets, representing data from 4 of the 5 species included in the analysis (Table S2).

\section{Functional Validation of a Role for Pafah1b1 in Alcohol Response in Mice}

The laboratory mouse is a powerful and efficient model system that has been used in many studies of alcohol drinking and alcohol response (Crabbe, 2014). Furthermore, previous work has shown strong conservation of Pafah1b1 in mouse and human (Hirotsune et al., 1998). Pafah1b1 has been shown to be coexpressed with GABA type-A receptor subunits (specifically Gabra1, Gabrb2, and Gabrg2), a family of neurochemical receptors associated with alcohol-use disorder (Mulligan et al., 2012). Hence, using mice to validate the role of Pafah1b1 in alcohol response is indicated. Mutations in the human gene PAFAH1B1 result in neuronal migration defects including lissencephaly, a smoothened cerebral cortex (Lo Nigro et al., 1997). Because several gene sets from the GeneWeaver analysis indicated differential regulation of this transcript in response to alcohol (Lewohl et al., 2000; Mulligan et al., 2011), we tested the hypothesis that perturbation of this gene affects acute behavioral and physiological response to alcohol. Other studies revealed increased Pafah1b1 abundance in mice selected for increased alcohol drinking so we also studied the preference for alcohol.

\section{Effects of Heterozygous Pafah1b1 Deletion on Alcohol Response}

As homozygous deficiency of Pafah1b1 results in embryonic lethality (Tokuoka et al., 2003), we tested heterozygous deficient mice (129S-Pafah1b1 $1^{\text {tm2.2Awb } / E j c) ~ a n d ~ c o m p a r e d ~ t h e m ~ t o ~ t h e i r ~}$ wild-type (WT) litter mate controls. In the present study we tested two cohorts of mice for alcohol response in two batteries of 
tests (Table S3). The first cohort received a low dose of ethanol, $1.25 \mathrm{~g} / \mathrm{kg}$ and the second cohort received two higher sedative doses of ethanol, 2.25 and $2.5 \mathrm{~g} / \mathrm{kg}$ at different ages. Cohort 1 comprised 18 heterozygous mutants (eight females and 10 males) and 22 WT mice ( 11 females and 11 males) at 18 weeks of age, and cohort 2 comprised 16 heterozygous mutants (10 females and six males) and 13 WT mice (10 females and three males) tested at both 18 weeks of age and again at 6-8 months of age (sufficiently removed from initial testing to avoid development of chronic tolerance). Cohort 1 was evaluated pre- and postethanol exposure using behavioral tests of locomotor activity in an open field, locomotor response on an accelerating rotarod, locomotor activity in a light-dark box and hypothermia. Cohort 2 was evaluated pre- and post-ethanol for hypothermia and lossof-righting reflex. Cohort 2 was then tested for alcohol preference over an increasing series of ethanol concentrations $(3,6,9,12$, and $15 \% \mathrm{v} / \mathrm{v}$ EtOH). Below are the results of the various tests.

\section{Hypothermia}

The magnitude of and tolerance to hypothermia in response to alcohol administration has been shown to be negatively correlated with withdrawal severity and is used as a predictive marker for susceptibility to ethanol dependence in genetic studies of rodents (Crabbe et al., 1983). We tested the hypothermic response of Pafah1b1 mutant mice to three different doses of alcohol: in cohort $1,1.25 \mathrm{~g} / \mathrm{kg}$, and in cohort $2,2.25 \mathrm{~g} / \mathrm{kg}$ and 6-8 weeks later, $2.5 \mathrm{~g} / \mathrm{kg}$ (Figure 3). Compared to WT male mice, Pafah1b1 mutant mice have a significantly decreased hypothermic response to 2.25 and $2.5 \mathrm{~g} / \mathrm{kg}$ alcohol but not to $1.25 \mathrm{~g} / \mathrm{kg} \mathrm{EtOH}$. There was a significant sex $\times$ genotype interaction $\left[F_{(2,23)}=18.2494, p<0.0001, q<0.0001\right]$ independent of dose $(2.25$ and $2.5 \mathrm{~g} / \mathrm{kg}$ were repeated measures of the same mice). At $2.25 \mathrm{~g} / \mathrm{kg}$, the body temperature of WT males decreased by $3.16 \pm \mathrm{SEM}=0.41$ degrees, whereas the body temperature of the Pafah $1 b 1$ mutant males decreased by only $1.98^{\circ} \mathrm{C} \pm \mathrm{SEM}=0.10$ degrees. The $2.5 \mathrm{~g} / \mathrm{kg}$ dose followed the same pattern. At $1.25 \mathrm{~g} / \mathrm{kg}$, the dose used in cohort 1 , there was only a univariate sex and ethanol treatment effect in all mice $\left[F_{(1,38)}=44.0213, p<0.0001, q=0.024\right.$;
$\left.F_{(1,38)}=6.9, p=0.0119, q=0.034\right]$. These results suggest that the heterozygous deficiency of Pafah1b1 in males results in an attenuated hypothermic response to higher (sedative) doses of ethanol.

\section{Ataxia-Motor Incoordination}

An increased overall sensitivity to acute alcohol may be protective against alcohol-use disorders (Schuckit, 1994). In this experiment ataxia, as a measure of alcohol sensitivity, was tested by measuring the latency to fall from a spinning rotarod (Figure 4A). Both control and Pafah1b1 mutant animals exhibited alcohol-induced ataxia on the rotarod at $1.25 \mathrm{~g} / \mathrm{kg}$ EtOH. There was a significant genotype $\times$ treatment interaction manner with crossover effects $\left[F_{(1,38)}=5.9395, p=0.0196\right.$, $q=0.041]$ such that Pafah $1 b 1$ mutant mice were less coordinated than WT mice at baseline but were more coordinated than WT mice after alcohol exposure.

\section{Sedation-Loss of Righting Reflex (LORR) and Duration of Loss of Righting Reflex (DLRR)}

Sensitivity to alcohol-induced sedation was tested by determining the time until the mouse lost its ability to right itself in a rotating chamber and the duration of time until this reflex was regained. Pafah 1 b1 mutant and litter-mate control mice from cohort 2 were given a sedative dose $2.5 \mathrm{~g} / \mathrm{kg}$ of ethanol (Figure $4 \mathrm{~B}$ ). There was a significant effect of sex and genotype $\left[F_{(2,23)}=11.5531\right.$, $p=0.0004, q=0.0001]$. There was no interaction of these factors on time to LORR in response to alcohol injection. Both Pafah1b1 mutant males and females had a lower LORR compared to WT mice, and females had a more rapid LORR than males. Similar effects were observed with time to recovery (DLRR). There was a significant effect of sex and genotype, but no interaction $\left[F_{(2,22)}=16.3215, p<0.0001, q=0.0001\right]$. The Pafah1b1 mutant mice recovered more quickly than the WT, and the females recovered more quickly than the males.

\section{Anxiety}

Prior research has shown a high rate of co-occurrence of alcohol-use disorders and anxiety disorders (Alegria et al., 2010). It is thought that the significant anxiolytic effects of alcohol
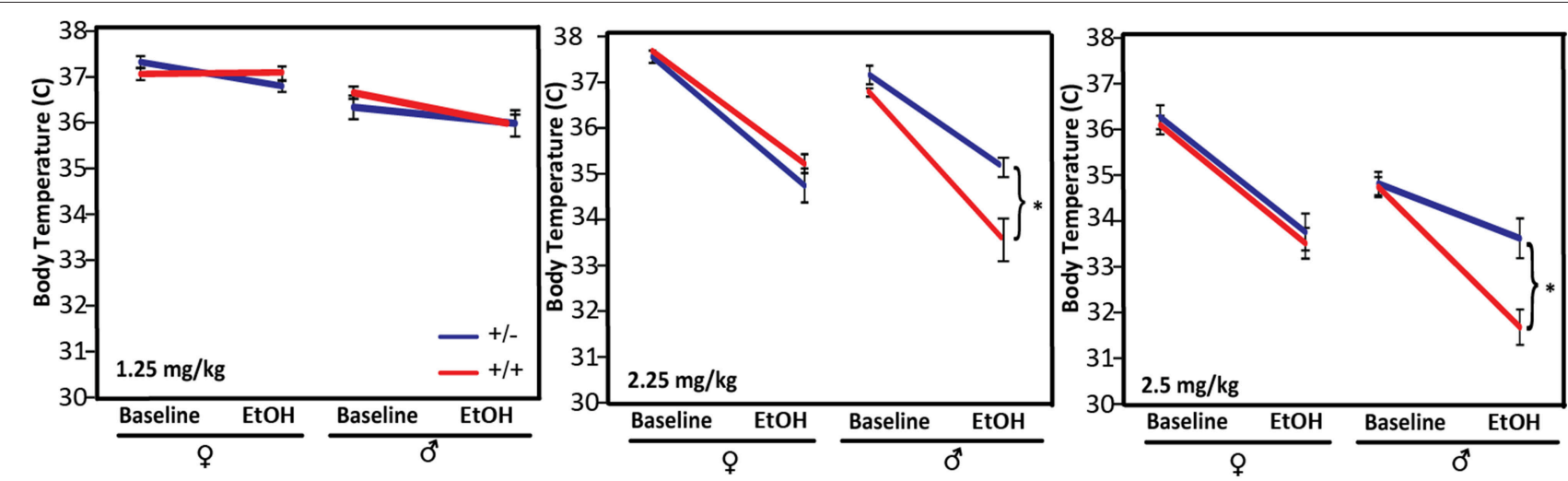

FIGURE 3 | Hypothermic response to different doses of ethanol, 1.25, 2.25, and $\mathbf{2 . 5} \mathbf{~ g / k g . ~ M e a n s ~} \pm$ SEM are depicted for $1.25 \mathrm{~g} / \mathrm{kg}\left(8 \pm q, 10 \pm \sigma^{7}, 11+/+q\right.$, $\left.11+/+\sigma^{x}\right), 2.25$, and $2.5 \mathrm{~g} / \mathrm{kg}\left(10 \pm q, 6 \pm \sigma^{x}, 10+/+q, 3+/+\sigma^{7}\right) .{ }^{*} p<0.05$. 


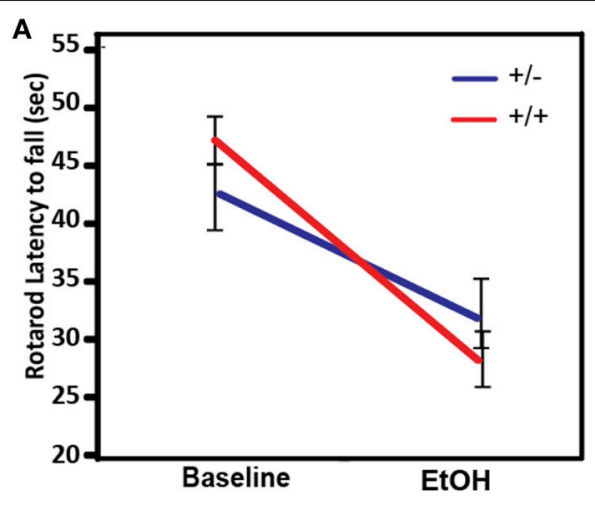

C
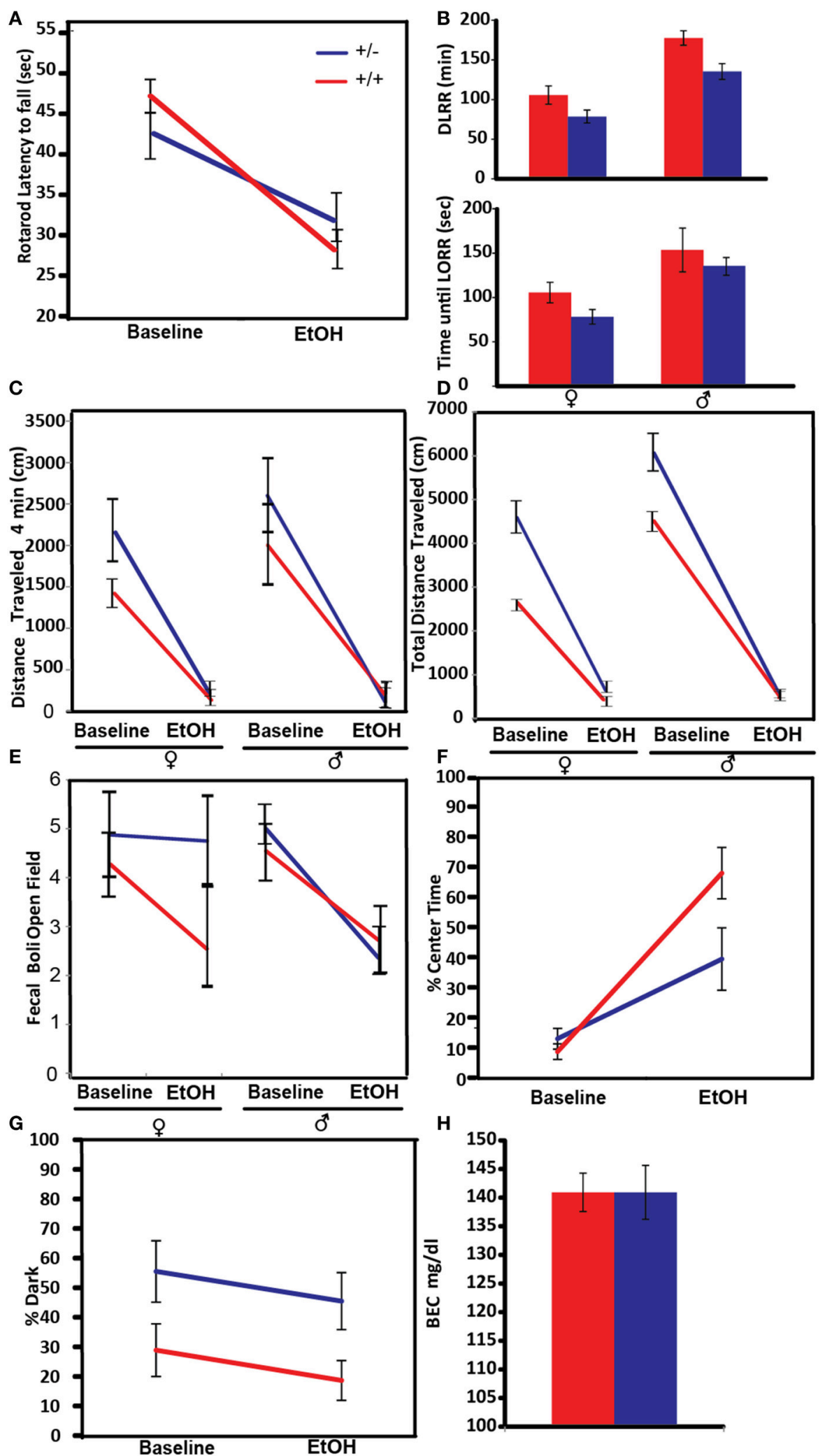

FIGURE 4 | Effects of Pafah1b1 heterozygous knock-out on the acute effects of alcohol on different behavioral and physiological traits. For each figure red indicates WT mice and blue indicates Pafah1b1 heterozygous mutant mice. (A) Ataxia was measured via latency to fall from an accelerating rotarod before and after a $1.25 \mathrm{~g} / \mathrm{kg} \mathrm{EtOH}$. (B) Sedation measured in response to $2.5 \mathrm{~g} / \mathrm{kg}$ EtOH as measured by time until LORR and time to recovery from LORR (DLRR). (C) Distance traveled after the first $4 \mathrm{~min}$ in a novel environment. (D) Total distance traveled in a novel environment. (E) Anxiety as measured by counts of fecal boli in the open field 
FIGURE 4 | Continued

after 20 min. (F) Percentage of time in the center of the open field as a measure of anxiety. (G) Percentage of time in the dark as a measure of anxiety. (H) Blood

ethanol concentration $1 \mathrm{~h}$ following $1.25 \mathrm{~g} / \mathrm{kg}$ dose of $\mathrm{EtOH}$. Means $\pm \mathrm{SEM}$ are depicted, $+/+n=22, \pm n=18$, except (B) (cohort 2, $+/+n=13, \pm n=16)$.

in patients with basal elevated anxiety may promote greater alcohol intake, which can lead to abuse (Robinson et al., 2009). We evaluated measures of anxiety-like behavior in Pafah1b1 mutant mice using measures of activity in a novel environment, defecation in the open field, percent time in the center of an open field and amount of time in the dark in a light-dark box.

\section{Locomotor Activity in the Open Field}

Anxiety-like behavior in the open field is often assessed by measuring exploratory behavior within the first few minutes of beginning the open field test (Prut and Belzung, 2003), an approach that we took in this experiment. The open field assay was done at baseline in a novel environment and the following day in response to $1.25 \mathrm{~g} / \mathrm{kg} \mathrm{EtOH}$. The distance traveled in the first $4 \mathrm{~min}$ (Figure 4C) showed a genotype $\times$ treatment effect $\left[F_{(1,36)}=6.31, p=0.016, q=0.041\right]$, where the Pafah1b1 mutants of both sexes traveled a greater distance compared to the WT mice. Total distance traveled in the open field (Figure 4D) showed a non-significant main effect of sex $\times$ treatment $\left[F_{(1,37)}=4.46, p=0.0415, q=0.441\right]$ and a significant univariate genotype effect $\left[F_{(1,37)}=4.5665\right.$, $p=0.0393, q=0.046]$ where the males traveled a further distance and the distance was much greater in Pafah1b1 mutants than in controls $(5176 \mathrm{~cm} \pm \mathrm{SEM}=945$ vs. $3432 \pm \mathrm{SEM}=336)$.

\section{Defecation in the Open Field}

The amount of defecation, as measured by the number of fecal boli in the open field at the conclusion of the open fie (Storey and Tibshirani, 2003) ld assay is used as an assay of anxiety. The fecal boli in the open field test results (Figure 4E) showed an anxiolytic effect of $1.25 \mathrm{~g} / \mathrm{kg} \mathrm{EtOH}\left[F_{(1,36)}=14.2132, p=0.0006\right.$, $q=0.024]$ with a non-significant sex $\times$ treatment interaction $\left[F_{(1,36)}=4.33502, p=0.0442, q=0.441\right]$ where female mice had a smaller change in response to ethanol, than the males. Genotype had no significant effect on the fecal boli response to alcohol $\left[F_{(1,36)}=2.3450, p=0.1344, q=0.063\right]$.

\section{Open Field Center Time}

We also tested anxiety via the percentage of time spent in the center of the open field box (Figure 4F). The percentage of time increased in response to $1.25 \mathrm{~g} / \mathrm{kg} \mathrm{EtOH}$ treatment $\left[F_{(1,38)}=34.0289, p<0.0001, q=0.024\right]$ with a significant genotype $\times$ treatment interaction $\left[F_{(1,38)}=4.9928, p=0.0314\right.$, $q=0.044]$, where Pafah $1 b 1$ mutants increased their center time in response to alcohol to a lesser degree than the litter-mate controls.

\section{Light Dark Preference}

The percent time spent in the dark compartment of the light-dark box was used as a measure of anxiety to the aversive light side (Figure 4G). In contrast to the observed increased exploratory activity in the open field, in the light-dark box mutant mice at baseline and post-ethanol injection tended to avoid the light to a greater extent ( $\sim 26 \%$ increase) compared to control mice, suggesting increased anxiety. The percentage of time in the dark showed a genotype effect $\left[F_{(1,37)}=6.3455, p=0.0162, q=\right.$ $0.041]$ but failed to show a treatment effect $\left[F_{(1,37)}=1.8333\right.$, $p=0.1840]$, as the percent time in the dark post-ethanol was not different from baseline. These results suggest the $1.25 \mathrm{~g} / \mathrm{kg}$ dose of $\mathrm{EtOH}$ was not sufficient enough to reduce the anxiety produced by the light side in either strain of mice.

\section{Blood Ethanol Concentrations}

The concentration of ethanol in the blood $1 \mathrm{~h}$ after injection with $1.25 \mathrm{~g} / \mathrm{kg}$ was not significantly different between the Pafah $1 \mathrm{bl}$ mutants and WT mice $\left[F_{(1,39)}=0.0105, p=0.9191,140.92\right.$ $\mathrm{mg} / \mathrm{dl} \pm \mathrm{SEM}=4.70$ vs. $141.50 \mathrm{mg} / \mathrm{dl} \pm \mathrm{SEM}=3.35$; Figure $4 \mathrm{H}]$.

\section{Effects of Heterozygous Deletion of Pafah1b1 on Alcohol Preference \\ Alcohol Preference}

Alcohol preference was measured using the two-bottle choice paradigm, in which one of two adjacent bottles contains ethanol and the other contains water. The experiment was carried out for 4 days at increasing concentrations of ethanol [3, 6, 9, 12, and 15\% (v/v)] (Figures 5A-F). Genotype $\times$ sex $\times$ dose had a significant effect on alcohol preference as expressed as a percentage of total fluid consumed $\left[F_{(4,22)}=3.2374, p=\right.$ 0.0312]. At low doses male Pafah $1 b 1$ mutant mice avoid ethanol compared to WT controls and prefer it at higher doses, whereas Pafah1b1 female mice trend toward preferring ethanol across all doses (Figures 5A,B). Alcohol intake in the two-bottle choice paradigm shows a trend toward a significant genotype $\times$ dose effect $\left[F_{(4,15)}=2.8029, p=0.064\right]$ with the Pafah1b1 mutants drinking more ethanol than the WT (Figure 5C) and a significant univariate sex effect $\left[F_{(1,18)}=5.9240, p=0.0256\right]$ where females drink more alcohol overall (Figure 5D). Total consumption of fluids over increasing doses shows a genotype $\times$ sex $\times$ dose interaction $\left[F_{(4,16)}=3.5023, p=0.0309\right]$, where female Pafah1b1 mutants drank the most ethanol over all doses and the wild type males drank the least (Figures 5E,F).

\section{DISCUSSION}

Through integration and analysis of genome-wide alcohol response experiments performed across species, curated from disparate sources and integrated in the interactive computational framework (GeneWeaver), multiple previously unknown alcohol related genes were identified, one of which Pafah1b1 was validated experimentally. Many others remain to be evaluated but are prioritized through convergence of evidence from among thousands of individual candidates. This integrative genomics analysis of diverse experimental data revealed a potential novel 

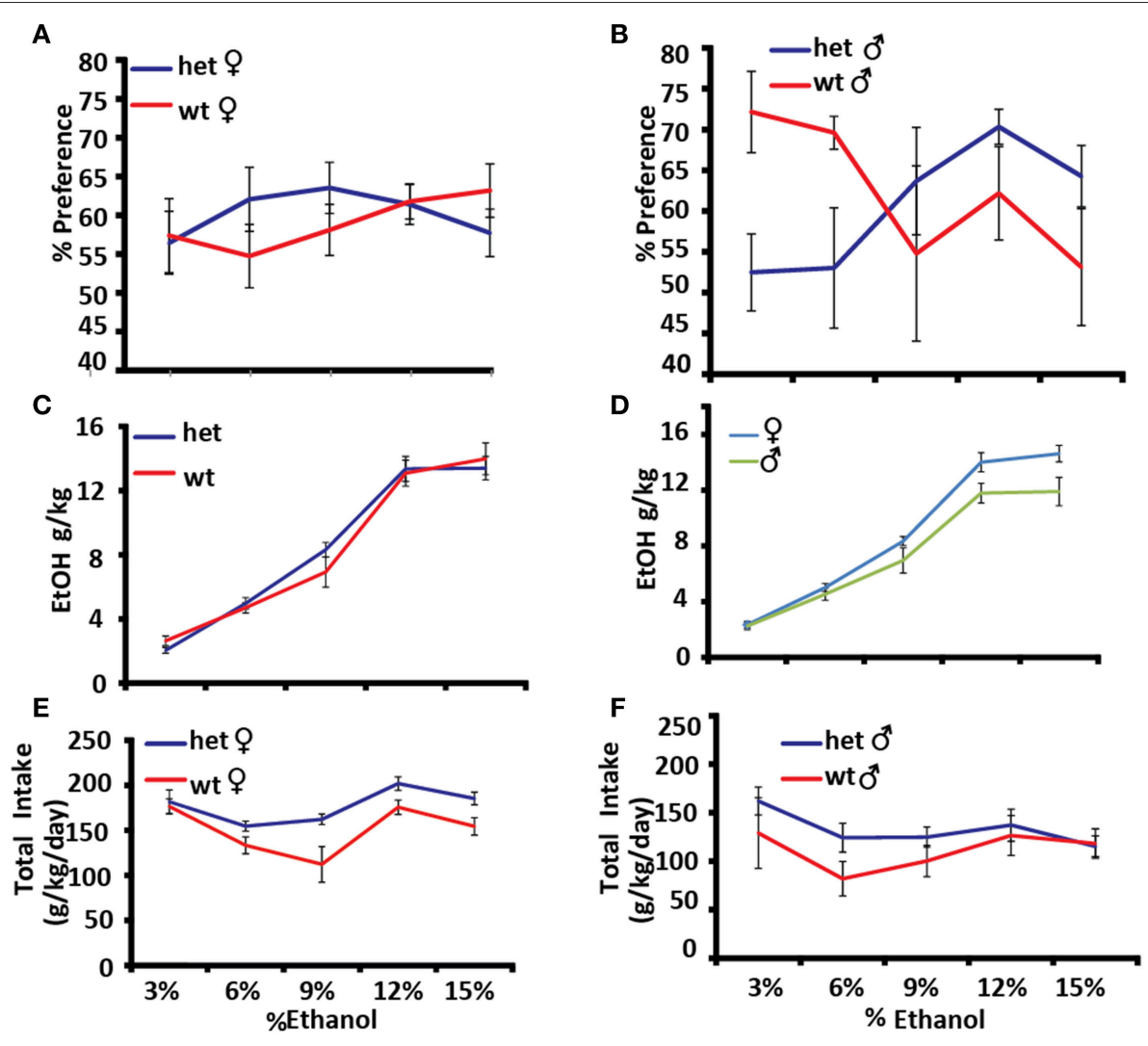

FIGURE 5 | Effects of Pafah1b1 heterozygous knock-out on alcohol preference. Alcohol preference was measured using the two-bottle choice paradigm for 4 days at increasing concentrations of ethanol $(\mathbf{3}, \mathbf{6}, \mathbf{9}, \mathbf{1 2}$, and $\mathbf{1 5} \%)$. (A) Alcohol preference expressed as a percentage of alcohol consumed divided by total fluid intake in females. (B) Alcohol preference expressed as a percentage of alcohol consumed divided by total fluid intake in males. (C) Alcohol consumed as g/kg in Pafah1b1 mutants and WT controls. (D) Alcohol consumed as g/kg in males and females. (E) Total fluid consumed as g/kg/day females. (F) Total fluid consumed as $\mathrm{g} / \mathrm{kg} /$ day males. Means \pm SEM are depicted $(\mathbf{A}, \mathbf{B})+/+n=13, \pm n=16$, male and female $(\mathbf{C}-\mathbf{F})+/+n=10, \pm n=13$, male and female.

role for Pafah1b1, a gene that had not been previously associated with alcohol-use disorder. This finding was confirmed using a knock-out mouse. The striking finding highlighted in Figure 1 is that the most highly connected genes, i.e., the genes present in the highest number of datasets from the alcohol-related studies analyzed in GeneWeaver, were not previously annotated to alcohol response in humans, as indicated in OMIM, or in mice, as indicated in the MP Ontology. Although we were surprised to find this, it is possible that the sparse annotation in OMIM and MP for the genes we identified from experimental data is the result of their essential roles in development, as in such cases a complete null would be lethal, as is the case with Pafah1b1 (Georgi et al., 2013). It is likely that the historical stringency of reporting for genome-wide experimental studies has been too high, resulting in a large number of false negative results that have escaped attention in initial analyses. The GeneWeaver system, and its underlying algorithms for gene set integration, addresses this issue by harmonizing diverse data, thereby reducing sparsity, and enabling integrative analysis to find convergent evidence for genes which may have weak but frequent experimental evidence for a role in disease. GeneWeaver captures and distills the experimental designs, analysis approaches, curation methods, and decision criteria imposed by experimenters to associate genes to biological concepts into a homogeneous data structure. We can represent the relations of biological entities, in this case genes, and the experimental constructs to which they are associated.

Mouse mutant models are a powerful system in which to evaluate the roles of genes in alcohol-related disorders. Several of the characteristics of alcohol-use disorder can be modeled directly or indirectly in mice, including alcohol preference, tolerance, and withdrawal (Crabbe, 2012). Alcohol preference as measured in a two-bottle choice paradigm is highly heritable, and is inversely correlated to withdrawal severity in rodents (Ford et al., 2011). The magnitude of and tolerance to hypothermia in response to alcohol administration has been shown to be negatively correlated with withdrawal severity and is used as a predictive marker for susceptibility to ethanol dependence in genetic studies of rodents (Crabbe et al., 1983). In the heterozygous Pafah1b1 mutant, we observed increased hypothermia in a dose-dependent manner in males (Figure 3) and a decreased preference for ethanol at low doses in the male mutants (Figure 5B). The female mutants showed no difference in hypothermic response (Figure 3) but exhibited a trend toward increased preference (Figure 5A). Pafah1b1 heterozygous mice showed increased basal anxiety (percentage of time in the dark at baseline) as well as hyperactivity. There was less response to 
alcohol observed in the center of the open field in the Pafah1b1 mutant mice (Figure 4F). Pafah1b1 heterozygous deficiency results in decreased anxiolysis in response to ethanol. The increased basal activity (hyperactivity) of the mutant mice in the open field, however, was restored to WT levels in response to ethanol, indicating that the $1.25 \mathrm{~g} / \mathrm{ml} \mathrm{EtOH}$ was sufficiently able to counteract the Pafah1b1 mutant's baseline hyperactivity. Both the sex and the genotype had effects on alcohol preference across the dose series. The most profound difference was the reduced preference for $3 \%$ seen in the male Pafah $1 b 1$ mutants that then crossed over at the higher doses (12-15\%) and became an increased preference. Overall, the female mutants also had slightly increased preference over WT across all doses. Pafah $1 b 1$ mutation had a strong impact on the sedative effects of alcohol. Compared to the wild-type littermate controls the mutants of both sexes were sedated much more rapidly than the controls (Figure 4B) but also recovered much more rapidly than the controls. This suggests that the mutants are more sensitive to the effects of ethanol but are also able to recover from them more rapidly.

Although, we know of no previous targeted studies of the role of this gene in alcoholism, such a role is plausible. The underlying mechanisms of alcohol dependence involve multiple signaling pathways and substantial neuroadaptive changes (Cui et al., 2013; Mahoney and Olmstead, 2013), which are first engaged during the acute response to alcohol. These acute responses such as hypothermia, LORR, and anxiolysis were impacted by a heterozygous Pafah1b1 mutant. The mechanism of Pafah1b1's action in alcohol response could function at several levels. First, Pafah1b1 has a role in synaptic integration (Hunt et al., 2012). Pafah1b1 mutations result in cortical, hippocampal and olfactory disorganization, reduced dendritic spine plasticity, impaired spatial learning, and coordination and social interactions (Paylor et al., 1999; Sudarov et al., 2013). The effects of alcohol on synaptic plasticity and neuromodulation are well-documented (reviewed in McCool, 2011). Second, Pafah1b1 plays a role in excitatory neurotransmission facilitated by dynamin (Sudarov et al., 2013). Most of the excitatory synaptic transmission in the nervous system is mediated by N-methyl-D-aspartate (NMDA) receptors. The inhibitory role of alcohol on the activity of the NMDA receptor channels has been extensively studied in numerous brain tissues (e.g., Yaka et al., 2003; Kolb et al., 2005) and variants of the receptor are associated with alcohol-use disorder (Kim et al., 2006; Pastor et al., 2009). Finally, Pafah1b1 plays a critical role in neuronal migration and cerebellar function (Cahana et al., 2001). Perturbation of this gene causes neuronal migration defects through cytoskeletal disruption, and its role in neuronal migration and synaptic function in cerebellar granule cells (Hunt et al., 2012) suggests a possible role for this gene in fetal alcohol syndrome (Jiang et al., 2008).

The scope of the literature on alcoholism, even restricted to the area of functional genomics, is vast. Through the use of integrated evidence and gene prioritization in GeneWeaver, it is possible to identify and prioritize research on novel roles for genes that are strongly implicated in phenomena such as alcohol-use disorders. GeneWeaver's data integration strategy is extensible to virtually any biological entity types in addition to the genes and gene products, providing facile integration and aggregation of convergent evidence. Many other applications of gene set integration exist (Bubier et al., 2015).

The increased emphasis and policy support for genomic data sharing will greatly accelerate the discovery of gene function in disease. The challenge remains, however, to extract meaningful biological signal from these data through the development of usable platforms and algorithms for interpretation of harmonized experimental results (Costello et al., 2013). The biomedical literature and the digital realm of biological data comprise a vast, yet sparsely annotated, and disparate collection of genomic data repositories, publication supplements, and curated experimental results. Accessing and integrating these extensive resources requires data integration and scalable computation and standardization. Truly making sense of the findings requires knowledge-discovery systems amenable to interrogation by functional biologists. GeneWeaver brings together convergent biological results from divergent species to identify highly supported but undiscovered roles of genes in disease, and to identify model organism resources and experimental strategies rapidly that are appropriate for confirmatory studies.

\section{AUTHOR CONTRIBUTIONS}

JB, EC Designed research, analyzed data. EB, ML, JJ, EC contributed analytic tools, JB, TW, EC performed research. JB, TW, JJ, ML, EB, and EC wrote the paper.

\section{FUNDING}

This project was supported by NIH R01 AA 18776 to EC. We gratefully acknowledge The Jackson Laboratory Scientific Services supported by NIH P30 CA034196.

\section{ACKNOWLEDGMENTS}

We thank the Reproductive Sciences Service for recovery of the cryopreserved floxed Pafah $1 b 1$ mouse. We gratefully acknowledge data-curation efforts by Dr. Vivek Philip and Amanda Ackovitz, and feedback on this manuscript by Stephen Sampson.

\section{SUPPLEMENTARY MATERIAL}

The Supplementary Material for this article can be found online at: http://journal.frontiersin.org/article/10.3389/fnbeh. 2016.00001

Figure S1 | Hi-resolution, Hierarchical Similarity Graph of experimentally derived alcohol-related genes sets, with the nodes labeled to identify the gene sets they contain.

Table S1 | The 32 gene set from genome wide functional genomic studies retrieved from GeneWeaver.org.

Table S2 | GeneWeaver gene sets containing Pafah1b1.

Table S3 | Testing Paradigm for the two cohorts. 


\section{REFERENCES}

Alegria, A. A., Hasin, D. S., Nunes, E. V., Liu, S. M., Davies, C., Grant, B. F., et al. (2010). Comorbidity of generalized anxiety disorder and substance use disorders: results from the National Epidemiologic Survey on Alcohol and Related Conditions. J. Clin. Psychiatry 71, 1187-1195. Quiz: 1252-1183. doi: 10.4088/jcp.09m05328gry

Amberger, J. S., Bocchini, C. A., Schiettecatte, F., Scott, A. F., and Hamosh, A. (2015). OMIM.org: online Mendelian Inheritance in Man (OMIM(R)), an online catalog of human genes and genetic disorders. Nucleic Acids Res. 43, D789-D798. doi: 10.1093/nar/gku1205

Bachmanov, A. A., Reed, D. R., Li, X., Li, S., Beauchamp, G. K., and Tordoff, M. G. (2002). Voluntary ethanol consumption by mice: genome-wide analysis of quantitative trait loci and their interactions in a C57BL/6ByJ x 129P3/J F2 intercross. Genome Res. 12, 1257-1268. doi: 10.1101/gr.129702

Baker, E. J., Jay, J. J., Bubier, J. A., Langston, M. A., and Chesler, E. J. (2012). GeneWeaver: a web-based system for integrative functional genomics. Nucleic Acids Res. 40, D1067-D1076. doi: 10.1093/nar/gkr968

Baker, E. J., Jay, J. J., Philip, V. M., Zhang, Y., Li, Z., Kirova, R., et al. (2009). Ontological Discovery Environment: a system for integrating gene-phenotype associations. Genomics 94, 377-387. doi: 10.1016/j.ygeno.2009.08.016

Bubier, J. A., and Chesler, E. J. (2012). Accelerating discovery for complex neurological and behavioral disorders through systems genetics and integrative genomics in the laboratory mouse. Neurotherapeutics $9,338-348$. doi: $10.1007 /$ s13311-012-0111-3

Bubier, J. A., Phillips, C. A., Langston, M. A., Baker, E. J., and Chesler, E. J. (2015). GeneWeaver: finding consilience in heterogeneous cross-species functional genomics data. Mamm. Genome. 26, 556-566. doi: 10.1007/s00335-015-9575-x

Cahana, A., Escamez, T., Nowakowski, R. S., Hayes, N. L., Giacobini, M., von Holst, A., et al. (2001). Targeted mutagenesis of Lis1 disrupts cortical development and LIS1 homodimerization. Proc. Natl. Acad. Sci. U.S.A. 98, 6429-6434. doi: 10.1073/pnas. 101122598

Costello, M., Michener, W., Gahegan, M., Zhang, Z., and Bourne, P. (2013). Biodiversity data should be published, cited, and peer reviewed. Trends Ecol. Evol. (Amst). 28, 454-461. doi: 10.1016/j.tree.2013.05.002

Crabbe, J. C. (2012). Translational behaviour-genetic studies of alcohol: are we there yet? Genes Brain Behav. 11, 375-386. doi: 10.1111/j.1601183X.2012.00798.x

Crabbe, J. C. (2014). Use of animal models of alcohol-related behavior. Handb. Clin. Neurol. 125, 71-86. doi: 10.1016/B978-0-444-62619-6.00005-7

Crabbe, J. C., Gray, D. K., Young, E. R., Janowsky, J. S., and Rigter, H. (1981). Initial sensitivity and tolerance to ethanol in mice: correlations among open field activity, hypothermia, and loss of righting reflex. Behav. Neural Biol. 33, 188-203. doi: 10.1016/S0163-1047(81)91625-3

Crabbe, J. C. Jr., Young, E. R., and Kosobud, A. (1983). Genetic correlations with ethanol withdrawal severity. Pharmacol. Biochem. Behav. 18(Suppl. 1), 541-547. doi: 10.1016/0091-3057(83)90233-2

Cui, C., Noronha, A., Morikawa, H., Alvarez, V. A., Stuber, G. D., Szumlinski, K. K., et al. (2013). New insights on neurobiological mechanisms underlying alcohol addiction. Neuropharmacology 67, 223-232. doi: 10.1016/j.neuropharm.2012.09.022

Davis, A. P., Murphy, C. G., Johnson, R., Lay, J. M., Lennon-Hopkins, K., SaraceniRichards, C., et al. (2013). The comparative toxicogenomics database: update 2013. Nucleic Acids Res. 41, D1104-D1114. doi: 10.1093/nar/gks994

Ford, M. M., Fretwell, A. M., Anacker, A. M., Crabbe, J. C., Mark, G. P., and Finn, D. A. (2011). The influence of selection for ethanol withdrawal severity on traits associated with ethanol self-administration and reinforcement. Alcohol. Clin. Exp. Res. 35, 326-337. doi: 10.1111/j.1530-0277.2010.01348.x

Gardner, D., Akil, H., Ascoli, G. A., Bowden, D. M., Bug, W., Donohue, D. E., et al. (2008). The neuroscience information framework: a data and knowledge environment for neuroscience. Neuroinformatics 6, 149-160. doi: 10.1007/s12021-008-9024-z

Georgi, B., Voight, B. F., and Bucan, M. (2013). From mouse to human: evolutionary genomics analysis of human orthologs of essential genes. PLoS Genet. 9:e1003484. doi: 10.1371/journal.pgen.1003484

Hamre, K. M., Goldowitz, D., Wilkinson, S., and Matthews, D. B. (2007). Screening for ENU-induced mutations in mice that result in aberrant ethanol-related phenotypes. Behav. Neurosci. 121, 665-678. doi: 10.1037/0735-7044.121.4.665
Henderson, N. D., Turri, M. G., DeFries, J. C., and Flint, J. (2004). QTL analysis of multiple behavioral measures of anxiety in mice. Behav. Genet. 34, 267-293. doi: 10.1023/B:BEGE.0000017872.25069.44

Hirotsune, S., Fleck, M. W., Gambello, M. J., Bix, G. J., Chen, A., Clark, G. D., et al. (1998). Graded reduction of Pafah1b1 (Lis1) activity results in neuronal migration defects and early embryonic lethality. Nat. Genet. 19, 333-339. doi: $10.1038 / 1221$

Hunt, R. F., Dinday, M. T., Hindle-Katel, W., and Baraban, S. C. (2012). LIS1 deficiency promotes dysfunctional synaptic integration of granule cells generated in the developing and adult dentate gyrus. J. Neurosci. 32, 12862-12875. doi: 10.1523/JNEUROSCI.1286-12.2012

Jiang, Y., Kumada, T., Cameron, D. B., and Komuro, H. (2008). Cerebellar granule cell migration and the effects of alcohol. Dev. Neurosci. 30, 7-23. doi: $10.1159 / 000109847$

Kim, J. H., Park, M., Yang, S. Y., Jeong, B. S., Yoo, H. J., Kim, J. W., et al. (2006). Association study of polymorphisms in N-methyl-D-aspartate receptor $2 \mathrm{~B}$ subunits (GRIN2B) gene with Korean alcoholism. Neurosci. Res. 56, 220-223. doi: 10.1016/j.neures.2006.06.013

Kolb, J. E., Trettel, J., and Levine, E. S. (2005). BDNF enhancement of postsynaptic NMDA receptors is blocked by ethanol. Synapse $55,52-57$. doi: 10.1002/syn.20090

Lewohl, J. M., Wang, L., Miles, M. F., Zhang, L., Dodd, P. R., and Harris, R. A. (2000). Gene expression in human alcoholism: microarray analysis of frontal cortex. Alcohol. Clin. Exp. Res. 24, 1873-1882. doi: 10.1111/j.15300277.2000.tb01993.x

Lim, J. P., Zou, M. E., Janak, P. H., and Messing, R. O. (2012). Responses to ethanol in C57BL/6 versus C57BL/6 x 129 hybrid mice. Brain Behav. 2, 22-31. doi: $10.1002 /$ brb3.29

Lo Nigro, C., Chong, C. S., Smith, A. C., Dobyns, W. B., Carrozzo, R., and Ledbetter, D. H. (1997). Point mutations and an intragenic deletion in LIS1, the lissencephaly causative gene in isolated lissencephaly sequence and MillerDieker syndrome. Hum. Mol. Genet. 6, 157-164. doi: 10.1093/hmg/6.2.157

Mahoney, M. K., and Olmstead, M. C. (2013). Neurobiology of an endophenotype: modeling the progression of alcohol addiction in rodents. Curr. Opin. Neurobiol. 23, 607-614. doi: 10.1016/j.conb.2013.03.006

McCool, B. A. (2011). Ethanol modulation of synaptic plasticity. Neuropharmacology 61, 1097-1108. doi: 10.1016/j.neuropharm.2010.12.028

McGary, K. L., Park, T. J., Woods, J. O., Cha, H. J., Wallingford, J. B., and Marcotte, E. M. (2010). Systematic discovery of nonobvious human disease models through orthologous phenotypes. Proc. Natl. Acad. Sci. U.S.A. 107, 6544-6549. doi: 10.1073/pnas.0910200107

Morozova, T. V., Anholt, R. R., and MacKay, T. F. (2007). Phenotypic and transcriptional response to selection for alcohol sensitivity in Drosophila melanogaster. Genome Biol. 8:R231. doi: 10.1186/gb-2007-8-10-r231

Mulligan, M. K., Ponomarev, I., Boehm, S. L. II, Owen, J. A., Levin, P. S., Berman, A. E., et al. (2008). Alcohol trait and transcriptional genomic analysis of C57BL/6 substrains. Genes Brain Behav. 7, 677-689. doi: 10.1111/j.1601183X.2008.00405.x

Mulligan, M. K., Rhodes, J. S., Crabbe, J. C., Mayfield, R. D., Harris, R. A., and Ponomarev, I. (2011). Molecular profiles of drinking alcohol to intoxication in C57BL/6J mice. Alcohol. Clin. Exp. Res. 35, 659-670. doi: 10.1111/j.15300277.2010.01384.x

Mulligan, M. K., Wang, X., Adler, A. L., Mozhui, K., Lu, L., and Williams, R. W. (2012). Complex control of GABA(A) receptor subunit mRNA expression: variation, covariation, and genetic regulation. PLoS ONE 7:e34586. doi: 10.1371/journal.pone.0034586

O'Gorman, S., Dagenais, N. A., Qian, M., and Marchuk, Y. (1997). ProtamineCre recombinase transgenes efficiently recombine target sequences in the male germ line of mice, but not in embryonic stem cells. Proc. Natl. Acad. Sci. U.S.A. 94, 14602-14607. doi: 10.1073/pnas.94.26.14602

Pastor, I. J., Laso, F. J., Ines, S., Marcos, M., and Gonzalez-Sarmiento, R. (2009). Genetic association between -93A/G polymorphism in the Fyn kinase gene and alcohol dependence in Spanish men. Europ. Psychiatry 24, 191-194. doi: 10.1016/j.eurpsy.2008.08.007

Paylor, R., Hirotsune, S., Gambello, M. J., Yuva-Paylor, L., Crawley, J. N., and Wynshaw-Boris, A. (1999). Impaired learning and motor behavior in heterozygous Pafah1b1 (Lis1) mutant mice. Learn. Mem. 6, 521-537. doi: 10.1101/lm.6.5.521 
Ponomarev, I., and Crabbe, J. C. (2002). A novel method to assess initial sensitivity and acute functional tolerance to hypnotic effects of ethanol. J. Pharmacol. Exp. Ther. 302, 257-263. doi: 10.1124/jpet.302.1.257

Prut, L., and Belzung, C. (2003). The open field as a paradigm to measure the effects of drugs on anxiety-like behaviors: a review. Eur. J. Pharmacol. 463, 3-33. doi: 10.1016/S0014-2999(03)01272-X

Robinson, J. A., Sareen, J., Cox, B. J., and Bolton, J. M. (2009). Correlates of self-medication for anxiety disorders: results from the National Epidemiolgic Survey on Alcohol and Related Conditions. J. Nerv. Ment. Dis. 197, 873-878. doi: 10.1097/NMD.0b013e3181c299c2

Rustay, N. R., Wahlsten, D., and Crabbe, J. C. (2003). Influence of task parameters on rotarod performance and sensitivity to ethanol in mice. Behav. Brain Res. 15, 237-249. doi: 10.1016/S0166-4328(02)00376-5

SAMHSA (2013). National Survey on Drug Use and Health (NSDUH). Rockville, MD: U.S. Department of Health and Human Services.

Schuckit, M. A. (1994). Low level of response to alcohol as a predictor of future alcoholism. Am. J. Psychiatry 151, 184-189. doi: 10.1176/ajp.151.2.184

Smith, C. L., and Eppig, J. T. (2009). The mammalian phenotype ontology: enabling robust annotation and comparative analysis. Wiley Interdiscip. Rev. Syst. Biol. Med. 1, 390-399. doi: 10.1002/wsbm.44

Storey, J. D., and Tibshirani, R. (2003). Statistical significance for genomewide studies. Proc. Natl. Acad. Sci. U.S.A. 100, 9440-9445. doi: $10.1073 /$ pnas. 1530509100

Sudarov, A., Gooden, F., Tseng, D., Gan, W. B., and Ross, M. E. (2013). Lis1 controls dynamics of neuronal filopodia and spines to impact synaptogenesis and social behaviour. EMBO Mol. Med. 5, 591-607. doi: 10.1002/emmm.2012 02106

Tokuoka, S. M., Ishii, S., Kawamura, N., Satoh, M., Shimada, A., Sasaki, S., et al. (2003). Involvement of platelet-activating factor and LIS1 in neuronal migration. Eur. J. Neurosci. 18, 563-570. doi: 10.1046/j.1460-9568.2003. 02778.x

Yaka, R., Phamluong, K., and Ron, D. (2003). Scaffolding of Fyn kinase to the NMDA receptor determines brain region sensitivity to ethanol. J. Neurosci. 23, 3623-3632.

Zhang, Y., Phillips, C. A., Rogers, G. L., Baker, E. J., Chesler, E. J., and Langston, M. A. (2014). On finding bicliques in bipartite graphs: a novel algorithm and its application to the integration of diverse biological data types. BMC Bioinformatics 15:110. doi: 10.1186/1471-2105-15-110

Conflict of Interest Statement: The authors declare that the research was conducted in the absence of any commercial or financial relationships that could be construed as a potential conflict of interest.

Copyright (c) 2016 Bubier, Wilcox, Jay, Langston, Baker and Chesler. This is an open-access article distributed under the terms of the Creative Commons Attribution License (CC BY). The use, distribution or reproduction in other forums is permitted, provided the original author(s) or licensor are credited and that the original publication in this journal is cited, in accordance with accepted academic practice. No use, distribution or reproduction is permitted which does not comply with these terms. 